\title{
CONTROL DEL ENTRENAMIENTO DEPORTIVO EN JUEGOS SURAMERICANOS MEDELLÍN 2010
}

\section{SPORTS TRAINING CONTROL IN THE SOUTHAMERICAN GAMES MEDELLIN 2010}

\author{
Diego A. Alzate S. ${ }^{1}$, Carlos F. Ayala Z.2, Luis G. Melo B. ${ }^{3}$
}

${ }^{1}$ Lic. Educación Física. Esp. Mgr. Facultad de Ciencias para la Salud, Universidad de Caldas, Avenida Paralela, Calle 48 esquina. Manizales, Caldas, Colombia. diego.alzate@ucaldas.edu.co 2 Lic. Educación Física. Esp. Mgr. Facultad de Ciencias para la Salud, Universidad de Caldas, Avenida Paralela, Calle 48 esquina. Manizales, Caldas, Colombia. federico.ayala@ ucaldas.edu.co ${ }^{3}$ Lic. Educación Física. Ph.D. Facultad de Ciencias para la Salud, Universidad de Caldas, Avenida Paralela, Calle 48 esquina. Manizales, Caldas, Colombia. luis.melo@ucaldas.edu.co

Rev. U.D.C.A Act. \& Div. Cient. 15 (Supl. Olimpismo): 87 - 95, 2012

\section{RESUMEN}

Con el objeto de determinar sí los entrenadores que asistieron a los deportistas de los Juegos Suramericanos Medellín 2010 aplicaban diferentes pruebas para hacer el control a los deportistas fue realizado un estudio cuantitativo, con alcance descriptivo, en el cual, fueron encuestados 93 entrenadores (86 hombres y 7 mujeres), con una edad media de 46,1 años ( $\pm 10,2)$, con un mínimo de 24 y una máxima de 79. La experiencia como entrenadores promedio fue de 20,5 $( \pm 9,8)$ años, entre 16,3 y 27,2 años, provenientes de 13 países en 28 deportes. La muestra fue intencional, puesto que no se conoció el dato exacto de los entrenadores asistentes al evento. La técnica utilizada fue la encuesta. Para el análisis de la información, se utilizó el programa Excel bajo Windows 2007, hallando que el $90,3 \%$ de los instructores consultados aplican diferentes pruebas de control, 79, entrenadores de campo, 45, de laboratorio y 41, ambas pruebas. Se presentó la tendencia que los deportistas entrenados por instructores de paises que aplicaban más pruebas de control (terreno y laboratorio), obtuvieron un mayor número de medallas.

Palabras clave: Pruebas de control, control bioquímico, campo, entrenamiento deportivo, entrenamiento físico.

\section{SUMMARY}

With the objective to determine if trainers that supported sportspeople in the South-American Games Medellin 2010, applied different tests in order to control sportsmen, a quantitative analysis was made which had a descriptive scope in which 93 trainers were surveyed ( 86 men and 7 women), aged in average 46.1 years ( \pm 10.2$)$ with a minimum age of 24 and a maximum of 79 . The average experience as trainer was of 20.5 years $( \pm 9.8)$; they came from 13 countries and were experts in 28 different sports. An intentional sample was taken, since the exact data of trainers attending the event was unknown. The technique used was the survey. In order to analyze the information an Excel program Windows 2007 was used. Results showed that $90.3 \%$ of the consulted coaches, applied different control tests, 79 trainers employed field control, 45 lab control and 41 both ways. A tendency, that the participants trained by coaches from countries where control tests were applied (field and lab) achieved a higher number of medals was detected.

Key words: Control test, biochemical control, test field, sports training, physical training.

\section{INTRODUCCIÓN}

Dentro de la investigación "Metodología del Entrenamiento Deportivo" realizada durante los Juegos Suramericanos Medellín 2010, tuvo como objetivo principal "identificar las características de la metodología del entrenamiento deportivo por parte de los entrenadores participantes en los Juegos Deportivos Suramericanos 2010”, a partir, del cual, se conoció el estado actual de las particularidades metodológicas de éste y que son tenidas en cuenta por los entrenadores suramericanos. Desde esa perspectiva, se abordó una, la que se refiere al "Control del Entrenamiento Deportivo" y cuyo objetivo fue "conocer los tipos de controles del entrenamiento utilizados por los entrenadores participantes en Juegos Suramericanos 2010", donde 
se pretendió conocer y analizar los diferentes modelos de control empleados.

Con base en lo anterior, se consideró que era pertinente preguntar a los entrenadores sobre el control del entrenamiento deportivo, para que, a partir de las respuestas obtenidas, se conocieran algunos modelos de control del entrenamiento suramericano y, a su vez, servir de apoyo y determinar cómo se pueden mejorar los procesos, ya que el rendimiento físico está condicionado por factores de tipo genético, estructural, fisiológico, biomecánico y psicológico, que redundan en la mejoría de las diferentes habilidades, técnicas y tácticas, características de cada actividad física y deportiva y que éstas, a su vez, puedan ser mejoradas o potenciadas, a través de procesos adaptativos, que denominamos entrenamiento deportivo (González, 1992; Weineck, 2005).

Después de hacer una revisión de las diferentes investigaciones que se han desarrollado en torno a aspectos relacionados con el control del entrenamiento deportivo (Arruza et al. 1996; Buceta, 1998; Ossorio, 2001; Barrios, 2002; Moya, 2004), se encontró que ninguna tenía relación con todas las características del control deportivo, pues pretendieron conocer aspectos puntuales y específicos. Algunas, como la de Calderón Montero et al. (2006), quienes al abordar el tema, plantean una serie de preguntas relacionadas con las herramientas que se requieren, los aspectos fisiológicos, técnicos, tácticos, psicológicos, la evaluación bilógica del deportista, la alta exigencia del entrenamiento deportivo y concluyó en reconocer la importancia de realizar pruebas de control, pues: "[...] se ha intentado demostrar la importancia que puede tener el control de determinados parámetros bilógicos en la planificación del entrenamiento. Naturalmente, no existe un único parámetro que nos da la información precisa [...]". Si al componente biológico se añaden otros condicionantes del rendimiento (técnicos, tácticos, psicológicos y otros), se comprenderá lo señalado.

También se encontró una investigación denominada "Lesiones y factores deportivos en futbolistas jóvenes", orientada por Olmedilla et al. (2006), en la que abordaron el control deportivo y su relación con las lesiones en el fútbol practicado por jóvenes; ellos atendieron aspectos cualitativos y cuantitativos del control, esencialmente, en la planificación y en el establecimiento adecuado de tiempo en el entrenamiento. Como conclusión, proponen que se lleven a cabo "programas de prevención, fundamentalmente basados en el control del estrés por parte de los jugadores".

En sintonía con lo anteriormente revisado, González (1992) y Weineck (2005) expresan que el entrenamiento deportivo es considerado un proceso, a través del cual, el entrenador propone una serie de estrategias en busca de un buen nivel por parte del deportista, con el objetivo de alcanzar altos logros y resultados en los periodos de competición, haciendo este desarrollo riguroso y sistematizado, donde los procedimientos de control son de suma importancia y cobran una relevancia en la calidad del proceso que se refiere. El control permite medir la eficacia en la preparación de los deportistas y la valoración objetiva de diferentes aspectos relacionados con su estado, su nivel, la ejecución de los planes y eficacia de la actividad competitiva (Serrato, 2008; Vasconcelos, 2000; Mishchenko \& Monogarov, 1995).

Por eso el entrenamiento deportivo, cuando es controlado desde diferentes estadios de crecimiento y de resultados competitivos de los deportistas, permite pensar y cuidar la salud del deportista, indicar, analizar, estudiar y proyectar el alcance de altos logros deportivos y, especialmente, del proceso llevado a cabo (Pancorbo \& Blanco, 1990).

Como se ha expuesto, se puede determinar que el realizar controles al entrenamiento deportivo es de suma importancia en los procesos con los deportistas, aunque es un tema poco abordado por los investigadores del área; pese a ello, es necesario conocer los diferentes aspectos cognitivos, físicos y sociales del deportista, durante su vida en el deporte.

En los últimos años, en la metodología del entrenamiento deportivo, se ha evidenciado un amplio progreso en el manejo de información objetiva, que luego se centrará en el control de la preparación del deportista, para orientar el proceso hacia la consecución de altos logros (Viru \& Viru, 2003; Weineck, 2005).

Para lograr que en el deporte de altos logros se obtengan resultados importantes, se hace necesario planificar el entrenamiento, teniendo en cuenta las diferentes variables que intervienen en el deportista, ya que a medida que se mejora la condición física y la técnica, aumenta la actividad fisiológica (González, 1992; Serrato, 2008; Platonov, 2001).

Control del entrenamiento deportivo: La eficacia del desarrollo de las distintas capacidades físicas depende de la utilización de medios y de métodos objetivos del control del desarrollo de las mismas; el control y la valoración objetiva de éstas en el deportista y las distintas posibilidades de los sistemas funcionales permite al entrenador y al deportista obtener una información precisa, que puede servir para tomar decisiones durante el proceso (Platonov \& Bulatova, 1991; Vasconcelos, 2000; Navarro \& Rivas,.2001).

Ramos (2001) considera que el control de la condición física es un elemento importante para establecer, en el momento oportuno, si se están cumpliendo los objetivos de la preparación y hace parte del proceso administrativo. Como lo 
plantea Zatsiorski (1989), "la recopilación de la información sobre el estado del objeto de dirección y la comparación de su valor real con el planificado se denomina control".

González (1992) y Platonov (1993) plantean que, actualmente, en la valoración de los procesos adaptativos derivados del entrenamiento deportivo, se deben trabajar en torno al desarrollo, al control y a la valoración del deportista, un conjunto de profesionales de diferentes áreas en un laboratorio o centro de valoración funcional, como médicos, profesionales en educación física, fisiólogos, biomecánicos, ingenieros, psicólogos, entre otros y esto, especialmente, en el área de entrenamiento deportivo. La aplicación sistemática de pruebas de valoración física, laboratorio y psicológicas permite la obtención de información valiosa sobre los procesos de adaptación; por eso, la información recolectada puede estar relacionada con:

- La capacidad funcional y los mecanismos de adaptación ante situaciones de solicitación máxima.

- El perfil o modelo de la respuesta funcional que caracteriza una actividad física o una prestación deportiva.

- La propia especificidad, validez y fiabilidad de la pruebas de valoración funcional.

- La participación de las diferentes vías metabólicas de la producción de la energía necesaria para la actividad o el rendimiento físico - deportivo (análisis bioenergético).

- Las diferencias en las respuestas fisiológicas, condicionada por variables biológicas, como la edad, el peso, el sexo, el deporte, el nivel de rendimiento y otras.

- El establecimiento de elementos objetivos o selección de individuos con capacidades físicas o coordinativas espaciales, para el alto rendimiento deportivo.

- La identificación y la medición de aspectos fisiológicos relevantes en el proceso de planificación, de programación, de realización y de control del entrenamiento, la definición de su intensidad, la valoración de los mecanismos y la dinámica de la respuesta adaptativa (Delgado, 2011).

En todo proceso de preparación física, sin importar la modalidad deportiva, es indispensable conocer el desarrollo de éste y los efectos que se producen en el deportista; para tal propósito, se hace necesaria la aplicación de diferentes test y procedimientos para controlar y, posteriormente, hacer evaluaciones del rendimiento del deportista, lo que permite establecer en qué nivel se encuentra y así organizar y reorganizar cargas, con el objetivo de llevarlo a un estado óptimo o de consecucion de altos logros deportivos (Bennassar, 2003).

Clases de control: En el control del entrenamiento deportivo, se utilizan, principalmente, el biomédico, el psicológico, el técnico, de carga, el competitivo y el pedagógico, mediante, los cuales, el rendimiento deportivo se puede controlar y evaluar por medio de pruebas pedagógicas (test deportivo motores) y biomédicas (fisiológicas y químicas) (Ramos, 2001; Platonov \& Bulatova, 1993).

Es así como el control bioquímico del entrenamiento puede ser considerado como un medio complejo, pero eficaz, para conseguir una correcta dirección del entrenamiento deportivo, mediante la utilización de la información obtenida en los test bioquímicos que, para llevarse a cabo, valoran diversos metabolitos y sustratos presentes en la sangre, en la orina, en la saliva o en el sudor. Los resultados obtenidos definen lo que está pasando en el deportista; es pertinente recordar siempre que el objetivo principal del control bioquímico del entrenamiento es ayudar a los entrenadores a conseguir el rendimiento máximo, evitando el sobreentrenamiento (Vasconcelos, 2000; Platonov, 2001).

El control bioquímico lo integran todas las mediciones bioquímicas realizadas después de la aplicación de cargas acumuladas, caracterizadas por su alta intensidad o volumen, con el fin de evaluar la capacidad de recuperación, el estado de adaptación o detectar tempranamente el sobre-entrenamiento u over reaching. También se pueden medir parámetros de química sanguínea, parámetros hematológicos y parámetros hormonales, que evalúen el estado anabólico o catabólico (relación testosterona cortisol) y otros establecidos en la literatura (Viru \& Viru, 2003; Platonov, 2001).

\section{MATERIALES Y MÉTODOS}

Para la recolección de la información, se hizo mediante una consulta bibliográfica exhaustiva, existente y actualizada; después, se reunió el grupo investigador y se determinaron los temas más pertinentes para la construcción de la encuesta; después y simultáneamente, se aplicó la misma a un grupo de entrenadores integrantes de las ligas deportivas del departamento de Caldas y se envió a revisión de juicio de expertos de ligas deportivas y universidades de Colombia; se ajustó con base en las sugerencias, para luego construir la encuesta definitiva, que se aplicó durante el proceso de recolección de la información. En los aspectos relacionados con el "Control del Entrenamiento Deportivo", se tuvieron en cuenta las siguientes preguntas: 1) Aplica pruebas (test) de control: SI - NO; 2) Qué tipos de test aplica: De terreno (Campo) - De laboratorio; 3) Cuáles son los test más utilizados; 4) Cuáles son los principales criterios de calidad que tiene en cuenta para seleccionar los test que aplica y, 5) Cuáles aspectos de la preparación evalúa usted con los test de control.

El tipo de estudio correspondió a un trabajo descriptivocuantitativo de corte transversal. Los participantes 
encuestados fueron 93 técnicos, seleccionados por conveniencia. Una vez contactados los técnicos en los diversos escenarios deportivos y lugares de descanso, se les informó sobre el objetivo de la investigación. Para el análisis de la información, se utilizó el programa Excel 2007, donde se clasificaron las diversas preguntas, para obtener los promedios respectivos. Con relación a las consideraciones bioéticas, la participación de los técnicos fue voluntaria, garantizando el anonimato en sus respuestas, la información que requirieran y la posibilidad de retirarse en el momento que consideraran conveniente. Una vez autorizaban resolver la encuesta, se les solicitó firmar el consentimiento informado. Se tuvo presente la Resolución 8430/1993, Art. 11, dado que la solución del instrumento no implicaba ningún riesgo para los participantes en el estudio.

\section{RESULTADOS Y DISCUSIÓN}

Características demográficas de la muestra evaluada: Fueron encuestados 93 entrenadores, provenientes de 13 de los 15 países participantes (86,6\%), en 28 modalidades deportivas (75,7\%); la edad media fue de 46,1 ( $\pm 10,2)$ años, con una mínimo de 24 y una máxima de 79 años; por sexos, hubo un marcado desequilibrio, con 84 hombres $(90,3 \%)$ y 7 mujeres $(9,7 \%)$, cuatro de ellas $(57,1 \%)$ entrenadoras de gimnasia rítmica con elementos.
Con base en los anteriores datos, se puede observar que existe una gran diferencia en edad entre los entrenadores que asistieron a los deportistas en los juegos suramericanos Medellín 2010, ya que al ser comparados con García (2000) y Graupera \& Ruiz-Pérez (2008) muestran hallazgos similares, pues la edad promedio de estas investigaciones son de 42,1 y 31,91 años, con un mínimo de 26 y 20, con un máximo de 62 y 54 años, para hombres y mujeres, respectivamente.

Aplica pruebas de control: Es pertinente tener en cuenta que en el proceso de planificación del entrenamiento deportivo, se debe considerar el control como un elemento importante (Ramos, 2001; Platonov \& Bulatova, 1993) y que es necesario realizar un diagnóstico inicial, cuyos controles posteriores deben ser supervisados por el entrenador (Gómez, 2004). Es por ello, que al momento de preguntar a los entrenadores si ellos aplican pruebas de control, el 90,3\% respondió que sí (Figura 1), asentando una gran preocupación el 9,7\% restante, ya que autores como Pérez (2008) y Morales (2005) reconocen la importancia del control del entrenamiento, como medio eficaz para determinar si se están cumpliendo los objetivos del proceso, "además de comprobar el nivel de preparación que el deportista obtiene en cada una de las etapas del proceso formativo" (Gómez, 2002a) y complementan Cañadas et al. (2010), quienes comentan que éstos ayudan no solo a la labor del entrenador sino que resulta ser una herramienta importante para analizar

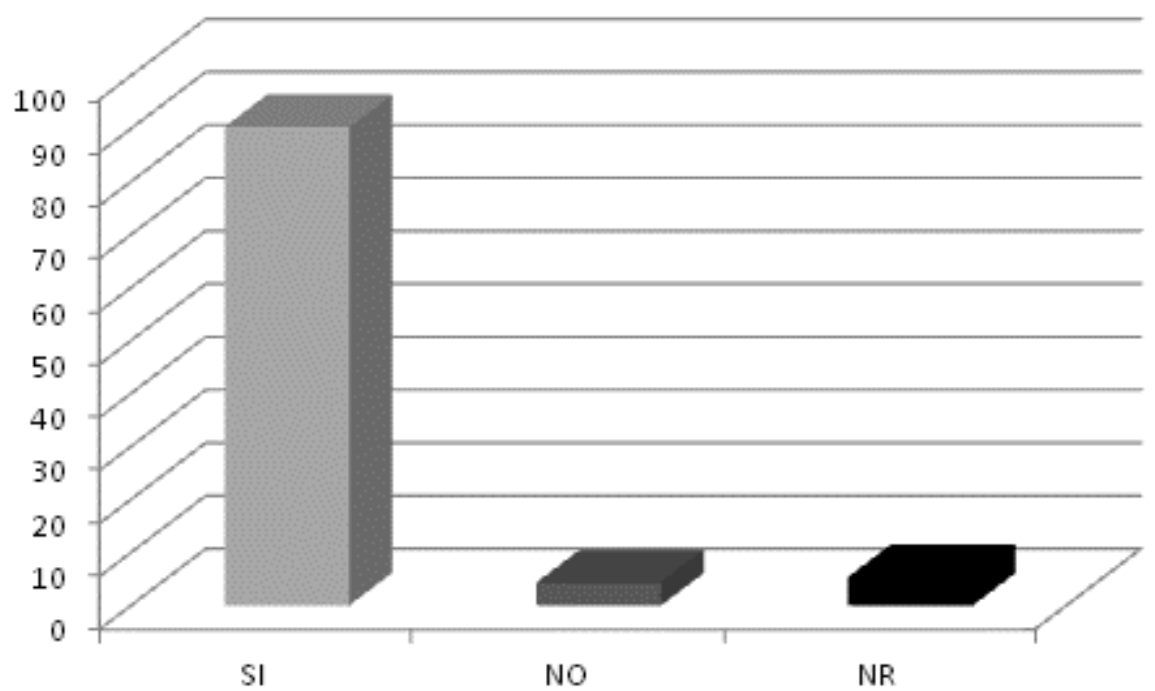

Figura 1. Aplicación de pruebas de control por parte de los entrenadores durante el periodo de preparación de los deportistas. 
el proceso de entrenamiento que se ha diseñado. Con base en las respuestas dadas, se puede determinar que algunos entrenadores no le hacen seguimiento a sus deportistas o peor aún, el proceso de selección no es el adecuado, ya sea por falta de conocimiento o por las caracteristicas de convocatoria de los deportistas, las cuales, muchas veces se hacen en días o en semanas previas a la competencia, especialmente, en deportes colectivos.

Lo anterior puede influir en el aseguramiento de un adecuado proceso de entrenamiento, ya que se supone que son entrenadores de alto rendimiento quienes van a dirigir seleccionados nacionales; también, puede indicar, que algunos entrenadores pueden ser contratados solo para el evento o para el campeonato próximo, reuniendo un grupo seleccionado u orientado por diversos entrenadores. Esta última situación la plantea Gómez (2002a y b), quien asegura que no es viable, ya que el entrenador no tiene tiempo para realizar un periodo de preparación y mucho menos de control o, lo que es peor aún, no conocen o no saben aplicar los diferentes test o no cuentan con el equipo adecuado y necesario.

Tipos de pruebas utilizadas: Según la referencia, las pruebas de laboratorio deben ser regularmente aplicadas durante el periodo de entrenamiento, para conocer cómo va el deportista en el proceso hacia la competencia y así tener evidencias objetivas (Serrato, 2008; Bennassar, 2003).

Como lo muestra la figura 2, las pruebas más utilizadas por los entrenadores son las de terreno, es decir, las que están relacionadas con la condición física y es, posiblemente, por la facilidad en su aplicación y que no requieren muchos implementos, espacios y elementos sofisticados para aplicarlas. Están a cargo directo del entrenador, por tener la formación específica del área y que permiten describir y determinar "el comportamiento del individuo en la prueba real. Por tanto, siempre es necesario recurrir a test específicos de campo de la prueba que realiza el individuo, que complementarán la información necesaria" (Rodríguez, 2001). En un segundo plano están las de laboratorio, claramente más complejas, ya que se debe tener un grupo interdisciplinario y el adecuado equipamiento (Díaz \& Romero, 2007) y que, según Escorcia (2011), "permite confirmar los cambios que experimenta el organismo y controlar la eficacia del entrenamiento respecto a una respuesta específica"; además, "las pruebas de laboratorio tienen una gran precisión para definir el rango de entrenamiento en función de la prueba deportiva "[...] pero tiene muchas limitaciones [...], debido al entorno tan controlado en temperatura, humedad" (Rodríguez, 2001); a ello también se le suma, el costo económico de los equipos para hacer las valoraciones,

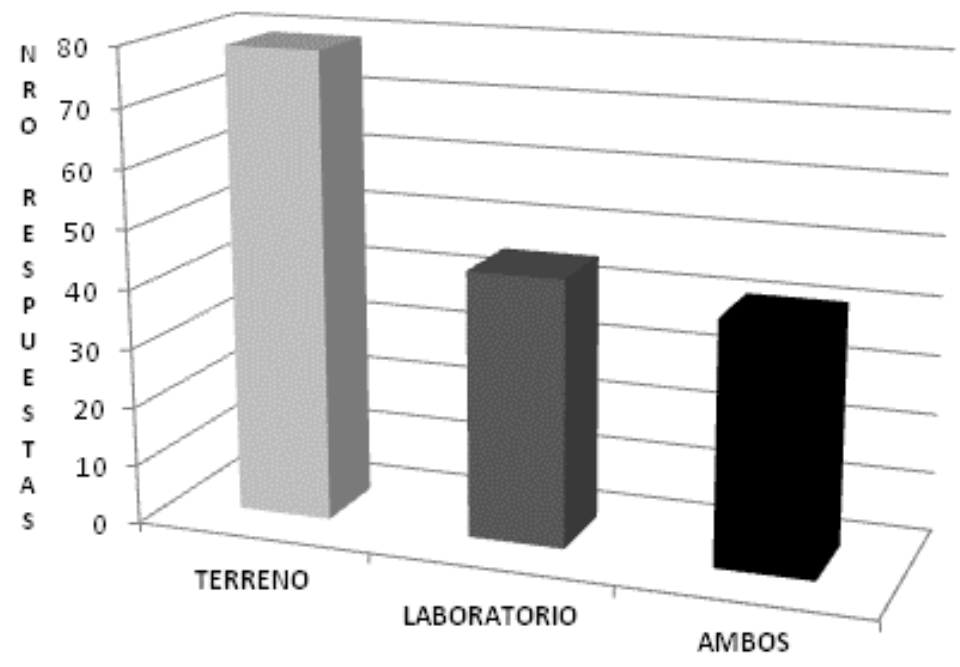

Figura 2. Grupos de pruebas utilizadas por los entrenadores. 
con lo cual, el grupo de entrenadores no cuenta o carece de un equipo humano calificado.

Así mismo, solo el $41 \%$ utiliza ambas pruebas, lo que permite inferir que, por lo menos, una parte de los entrenadores encuestados son conscientes de la importancia de la aplicación de pruebas de terreno y de laboratorio; igualmente, poseen los medios y los equipos del área necesarios para tener un mejor control del entrenamiento de los deportistas.

Lo anterior, se relaciona con las medallas obtenidas, pues se evidenció que el grupo de entrenadores que aplican ambas pruebas tuvieron un mayor porcentaje de medallas de oro, que aquellos que solo aplicaron una sola prueba, lo que demuestra, que una buena relación entre el tener un equipo interdisciplinario apropiado, con la capacidad de aplicar pruebas y hacer el análisis correcto para identificar las variables que están interviniendo favorable o desfavorablemente en el proceso, es más factible organizar la planificación y adecuarla a los deportistas, en relación a los propósitos planteados al inicio del proceso.

En conclusión es muy importante llevar a cabo controles del entrenamiento deportivo en diferentes deportes y modalidades $\mathrm{y}$ es pertinente tener en cuenta las características y las especificaciones de éstos, pues pueden variar, a causa de las mismas e, incluso, entre ellos, aunque los tipos de pruebas se complementan: "es conveniente cuidar meticulosamente la realización de las pruebas de campo, con unos métodos de evaluación estrictos, ya que se optimizarán los resultados y reflejarán mejor las características a investigar en los atletas" (Da Silva et al. 2004).

Aspectos de la preparación evaluados con los test: Con relación a la figura 3 , se puede determinar que no todos los entrenadores aplican test deportivos; además, no cuentan con un equipo interdisciplinario en el área de control que les pueda mostrar, de forma más amplia y científica, del cómo se hallan en rendimiento sus deportistas y así hacer las modificaciones necesarias (Platonov \& Bulatova, 1993; Weineck, 2005). La encuesta aplicada indicó que sólo 18 entrenadores aplican las cuatro áreas: físicas, psicológicas, bilógicas y nutricionales que, según Gómez (2002a), "el empleo de un sistema de entrenamiento, controlado mediante diversos indicadores, permite en cierta forma garantizar resultados competitivos", los demás, utilizan dos pruebas o menos, de lo que se puede inferir que no cuentan con el apoyo y los recursos suficientes para contratar un equipo completo o, tal vez, algunos entrenadores no lo consideran necesario.

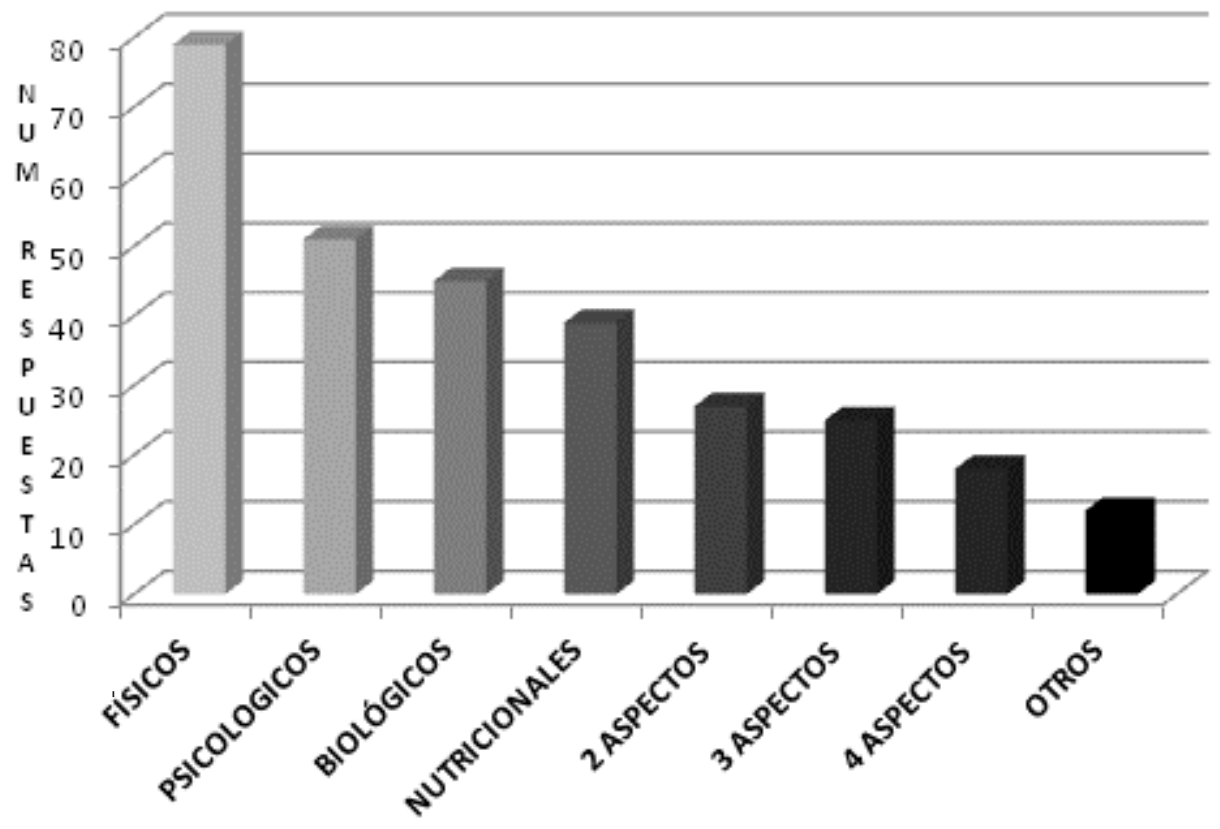

Figura 3. Aspectos de la preparación evaluados con las pruebas. 


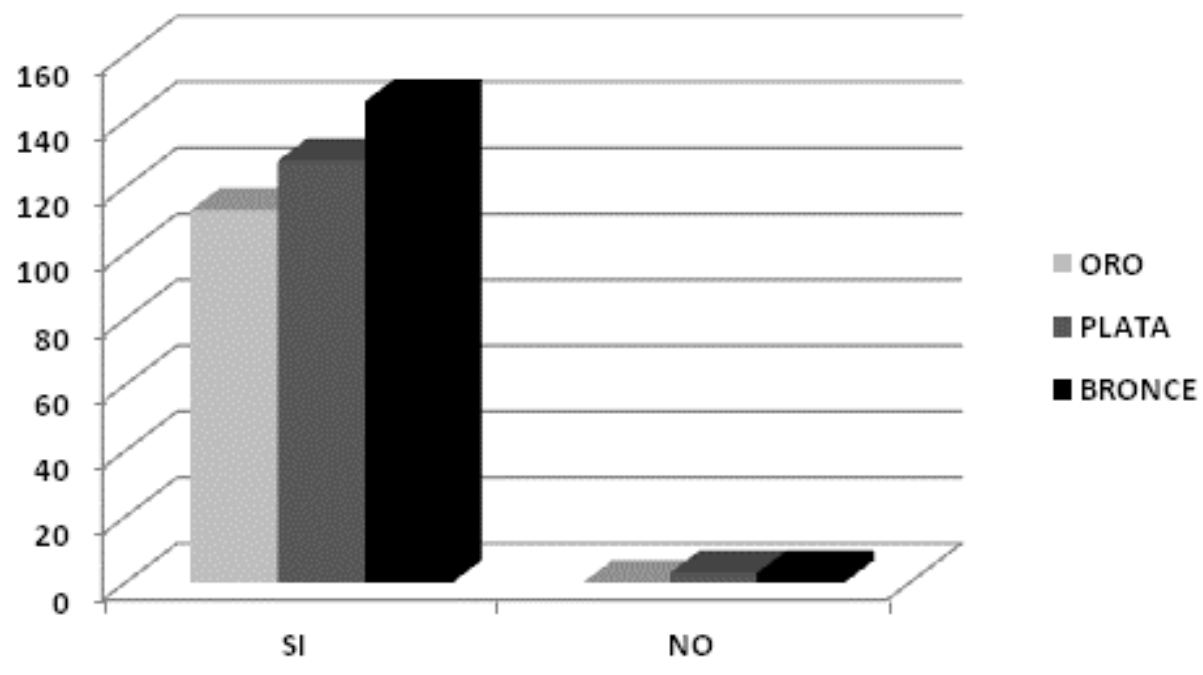

Figura 4. Medallería obtenida por parte de los encuestados según aplicación de pruebas.

Con relación al aspecto técnico, se presentaron siete respuestas, seguido de lo táctico y de volumen, con una respuesta cada uno. De la totalidad de los encuestados solo cuatro entrenadores manifestaron no aplicar ningún test de control para sus deportistas, ellos sumaron un total de tres medallas de plata, tres de bronce y ninguna de oro (Figura 4). El análisis de los datos refleja una tendencia que, aquellos entrenadores que aplican diferentes test de control a sus deportistas, con el propósito de hacerles seguimiento, obtuvieron un número representativo de medallas: 113, de oro; 128 , de plata y 146, de bronce (Figura 4).

Agradecimientos: Los autores agradecen a la Vicerrectoría de Investigaciones y Posgrados de la Universidad de Caldas, por auspiciar el desarrollo de la investigación "Metodología del entrenamiento deportivo en Juegos Suramericanos Medellín 2010", de la cual, hace parte el presente artículo; además agradecen a INDEPORTES Antioquia, por acreditar a los investigadores para ingresar a los diferentes espacios deportivos, para realizar la recolección de la información. Conflictos de interés: El manuscrito fue preparado y revisado con la participación de todos los autores, quienes declaramos que no existe ningún conflicto de intereses que ponga en riesgo la validez de los resultados presentados.

\section{BIBLIOGRAFÍA}

1. ARRUZA, J.; ALZATE, R.; VALENCIA, J. 1996. Esfuerzo percibido y frecuencia cardiaca: el control de la intensidad de los esfuerzos en el entrenamiento de judo. Rev. Psicol. Deporte. 9:29-40.

2. BARRIOS, D.R. 2002. Consideraciones sobre métodos de control psicológico en el entrenamiento de resistencia. Buenos Aires. Disponible desde Internet en: http://www.efdeportes.com/efd45/resist.htm (con acceso 24/04/2012).

3. BENNASSAR TORRANDELL, M. 2003. Manual de Educación Física y Deportes: Técnicas y actividades prácticas. Ed. Océano: Barcelona (España). 607p.

4. BUCETA, J.M. 1998. Psicología del entrenamiento deportivo. Dykinson: Madrid, 462 p.

5. CALDERÓN MONTERO, F.J.; BENITO PEINADO, P.J.; MELÉNDEZ ORTEGA, A.; GONZÁLES GROSS, M. 2006. Control Biológico del entrenamiento de resistencia. Rev. Int. Cienc. Deporte (España). 2(2):65-87. 
6. CAÑADAS, M.; IBÁÑEZ, S.J.; GARCÍA, J.; PAREJO, I.; FEU, S. 2010. Importancia de la planificación en el entrenamiento deportivo: análisis del proceso de entrenamiento en minibasket. España 2010. Disponible dese Internet en: http:/www.wanceulen.com/ revista/PDF/n7/7-7-planificacion-entrenamiento-minibasket.pdf (con acceso 09/03/2012).

7. DA SILVA, M.E.; NUÑEZ, A.V.; VAAMONDE, D.M.; IBNZIATEN, H.A.; VIANA, B.; GÓMEZ, J.R.; LANCHO, J.R. 2004. Diferencia en la Capacidad de Salto en el Laboratorio y en la Cancha. Un estudio transversal. Córdoba Disponible dese Internet en: http://www.deportesaciclicos.com/verart. asp? categoryld $=320 \&$ file $=$ capac_salto.pdf $\quad$ (con acceso 22/04/2012).

8. DELGADO, M.A. 2011. Evaluación funcional. Disponible desde Internet en: http://efisica-517-marcodelgado. blogspot.com/2011/10/valoracion-funcional.html (con acceso 23/04/2012).

9. DÍAZ B., P.L.; ROMERO E., R. 2007. Deporte de Alto Rendimiento: Gerencia, ciencia y tecnología. Tunja. Indeportes Boyacá. 388p.

10. ESCORCIA C., B. 2011. Control bioquímico del entrenamiento deportivo. La Habana. Disponible desde Internet en: http://portal.inder.cu/index.php/ recursos-informacionales/ato-ren/10275-controlbioquimico-del-entrenamiento-deportivo (con acceso marzo 08/03/2012).

11. GARCÍA, U.F. 2000. Entrenadores y Burnout. Buenos Aires. Disponible desde Internet en: http://www. efdeportes.com/efd28/burnout.htm (con acceso 22/04/2012).

12. GÓMEZ C., P. 2002a. Control del proceso de entrenamiento deportivo de alta competencia. Buenos Aires. Disponible desde Internet en: http:// www.efdeportes.com/efd55/entren.htm (con acceso 09/03/2012).

13. GÓMEZ C., P. 2002b. Alcances obtenidos mediante la planificación y control del proceso de entrenamiento deportivo en el taekwondo. (Estudio de casos). Buenos Aires Disponible desde Internet en: http:// ebookbrowse.com/alcances-obtenidos-mediantela-planificacion-y-control-pdf-d272036787 (con acceso 08/03/2012).
14. GÓMEZ, C., P. 2004. Planificación y programación del entrenamiento deportivo en taekwondo. Buenos Aires. Disponible desde Internet en: http://www.efdeportes. com/efd73/tkwd.htm (con acceso 08/03/2012).

15. GONZÁLEZ, G.J. 1992 Fisiología de la Actividad Física y el Deporte. Ed. Interamericana Madrid (España). 382p.

16. GRAUPERA, J.L.; RUIZ-PÉREZ, L.M. 2008. Estilos de Aprendizaje Experiencial em Entrenadores Deportivos Españoles. España. Disponible desde Internet en: www.5congreso-aecd.unileon.es/comunicaciones/ grauperaestilos.pdf (con acceso 22/04/2012).

17. MORALES A., A. 2005. La preparación del deportista: control y evaluación de la unidad de entrenamiento. Buenos Aires. Disponible desde Internet en: http:// www.efdeportes.com/efd86/control.htm (con acceso 08/03/2012).

18. MOYA MORALES, J.M. 2004. La percepción subjetiva del esfuerzo como parte de la evaluación de la intensidad del entrenamiento. Buenos Aires. Disponible desde Internet en: http://www.efdeportes.com/efd73/ percep.htm (con acceso 23/04/2012).

19. NAVARRO, F.; RIVAS, A. 2001. Planificación y Control del Entrenamiento en Natación. Ed. Gymnos: Madrid (España). 153p.

20. OLMEDILLA ZAFRA, A.; ANDREU ÁLVAREZ, M.; ABENZA CANO, L.; ORTÍN MONTERO, F.; BLAS REDONDO, A.; BLAS REDONDO, A. 2006. Lesiones y factores deportivos en futbolistas Jóvenes. Rev. Cultura, Ciencia y Deporte. (España). 2(5):59-66.

21. OSSORIO L., D. 2001. La influencia motivadora del entrenamiento integrado sobre la percepción del esfuerzo en el entrenamiento aeróbico. Buenos Aires. Disponible desde Internet en: http://www.efdeportes. com/efd41/infl.htm (con acceso 24/04/2012).

22. PANCORBO, A.; BLANCO. J. 1990. Consideraciones sobre le entrenamiento deportivo en la niñez y la adolescencia. Arch. Med. Deporte. (Cuba). 7(27):309-314.

23. PÉREZ G., E. 2008. Las pruebas o test en el deporte. Buenos Aires. Disponible desde Internet en: http:// www.efdeportes.com/efd126/las-pruebas-o-tests-enel-deporte.htm (con acceso 08/03/2012). 
24. PLATONOV, V.N. 2001. Teoría General del Entrenamiento Deportivo Olímpico. Ed. Paidotribo: Barcelona (España). 686p.

25. PLATONOV, V. 1993. El entrenamiento deportivo. Teoría y Metodología. Ed. Paidotribo. (España). 322p.

26. PLATONOV, V.N.; BULATOVA, M.M. 1993. La Preparación Física. Ed. Paidotribo. Barcelona (España). 313p.

27. RAMOS B., S. 2001. Entrenamiento de la Condición Física. Ed. Kinesis: Armenia (Colombia). 180p.

28. RODRÍGUEZ H., J.F. 2001. La Evaluación Fisiológica del Deportista de Alto Nivel. España. Disponible dese Internet en: www.ugr.es/ jhuertas/ EvaluacionFisiologica/Introduccion.htm (con acceso 22/04/2012).

29. MISHCHENKO, V.S.; MONOGAROV, V.D. 1995. Fisiología del deportista. Ed. Paidotribo: Barcelona (España). 328p.
30. SERRATO R. M. 2008. Medicina del deporte. Ed. Universidad del Rosario. Bogotá D.C. (Colombia). 484p.

31. VASCONCELOS R., A. 2000. Planificación y Organización del Entrenamiento Deportivo. Ed. Paidotribo: Barcelona (España). 198p.

32. VIRU, A.; VIRU, M. 2003. Análisis y Control del Rendimiento Deportivo. Ed. Paidotribo: Barcelona (España). 286p.

33. WEINECK, J. 2005. Entrenamiento Total. Ed Paidotribo: Barcelona (España). 688p.

34. ZATSIORSKI, V.M. 1989. Metrología Deportiva. Libro de texto. Ed. Pueblo y Educación: Habana (Cuba). 309p.

Recibido: Marzo 3 de 2012

Aceptado: Mayo 10 de 2012 